NASA Technical Memorandum 102138

AIAA-89-2669

\title{
High Speed Corner and Gap-Seal Computations Using an LU-SGS Scheme
}

William J. Coirier

Lewis Research Center

Cleveland, Ohio

(HASA-TH-102138) HIGH SPEED CORHBR AYD

GAP-SEAL COHPUTATIORS OSING AN LU-SGS SCBEHB

(NASA., Levis Research Center) 16 pCSCL 20D
N89-27103

Unclas 0217620

Prepared for the

25th Joint Propulsion Conference

cosponsored by the AIAA, ASME, SAE, and ASEE

Monterey, California, July 10-12, 1989 


\title{
HIGH SPEED CORNER AND GAP SEAL COMPUTATIONS USING AN LU-SGS SCHEME
}

\author{
William J. Coirier ${ }^{\dagger}$ \\ NASA Lewis Research Center \\ Cleveland, Ohio 44135
}

\begin{abstract}
The hybrid LU-SGS (Lower-Upper Symmetric Gauss-Seidel) algorithm has been added to a widely used series of $2 \mathrm{D} / 3 \mathrm{D}$ Euler/Navier-Stokes solvers and has been demonstrated for a particular class of highspeed flows. A limited study has been conducted to compare the hybrid LU-SGS for approximate Newton iteration and diagonalized Beam-Warming (DBW) schemes on a work and convergence history basis. This study showed that for the cases considered, the hybrid LU-SGS algorithm is more efficient and easier to implement than the DBW scheme originally present in the code. The code has been validated for the hypersonic flow through two mutually perpendicular flat plates and then used to investigate the flow field in and around a simplified scramjet module gap seal configuration. Due to the similarities, the gap seal flow was compared to hypersonic corner flow at the same freestream conditions and Reynolds number.
\end{abstract}

\section{Introduction}

The hybrid LU-SGS (Lower-Upper Symmetric Gauss-Seidel) scheme has been added to the PARC $2 \mathrm{D} / 3 \mathrm{D}$ series of Euler/Navier-Stokes solvers. The PARC codes are based upon a central finite-difference scheme with fourth and second difference dissipative terms. The PARC codes originated as the AIR 2D/3D solvers by Pulliam (Ref. 1), which used the ADI algorithm presented by Beam and Warming (Ref. 2). To increase the computational efficiency and to reduce storage requirements, Pulliam (Ref. 3) diagonalized the block implicit operators of the original Beam-Warming ADI algorithm, resulting in the ARC 2D/3D codes. Cooper (Ref. 4) further modified the ARC codes to be used in a production environment for solving the propulsion oriented problems at the Arnold Engineering Development Center, resulting in the PARC codes. The PARC codes are well validated and accepted and are in wide use by the aerodynamics community.

$\dagger$ Aerospace Engineer, Computational Methods Br. Member AIAA.

Copyright $C 1989$ by the American Institute of Aeronautics and Astronautics. Inc. No copyright is asserted in the United States under Title 17. U.S Code. The U.S. Government has a royalty-free license to exercise all rights under the copyright clatimed herein for Governmental purposes

All other rights are reserved by the copyright owner.
Through the use of a unique method of grid patching and the segmented application of boundary conditions, the PARC codes are very useful in solving wide varieties of flow problems. A full description of the PARC codes and their implementation of the diagonalized BeamWarming algorithm can be found in Reference 4 .

In an attempt to further advance the practicality of these solvers, the present work investigates the addition of a relatively new algorithm to the codes. An LU implicit scheme, proposed by Jameson and Turkel (Ref. 5) and improved upon and demonstrated for the Euler equations by Jameson and Yoon (Ref. 6) was used in conjunction with a multigrid strategy for the Euler equations by Jameson and Yoon (Ref. 7). From this scheme, the LU-SGS (also referred to as the LUSSOR) scheme was developed and applied by Yoon and Jameson in References 8 and 9 for solutions to the Euler and Navier-Stokes equations. This scheme was shown to be very efficient and practical for the solution of the Navier-Stokes equations coupled in a fully implicit manner to the chemical transport equations by Shuen and Yoon (Ref. 10) in two dimensions and by Yu, Tsai and Shuen (Ref. 11) in three dimensions. Recently, this scheme was used by Park and Yoon (Ref. 12) to compute two dimensional flow in chemical and thermal non-equilibrium around a blunt body. This scheme has clear advantages when compared to others for implicitly solving fairly large systems of transport equations since it has the operational count and storage requirements of an explicit scheme, but still solves the equations implicitly and fully coupled. The following sections will show the derivation of the LU-SGS scheme, compare the LU-SGS and diagonalized Beam-Warming schemes in terms of convergence history on a work basis and use the LU-SGS scheme to analyze hypersonic corner and gap seal flowfields.

\section{Analysis}

The PARC2D/3D codes solve the Reynold's averaged Navier-Stokes equations in a generalized curvilinear coordinate system. The Navier-Stokes equations can be written in divergence form as 


$$
\frac{\partial q}{\partial t}+\frac{\partial\left(E-E_{v}\right)}{\partial \xi}+\frac{\partial\left(F-F_{v}\right)}{\partial \eta}+\frac{\partial\left(G-G_{v}\right)}{\partial \zeta}=0
$$

where the flux variables $q$ and a generalized inviscid flux vector, $\hat{E}$, can be expressed as

$$
q=J^{-1}\left(\begin{array}{c}
\rho \\
\rho u \\
\rho v \\
\rho w \\
E_{t}
\end{array}\right), \quad \hat{E}=J^{-1}\left(\begin{array}{c}
\rho u_{c} \\
\rho u u_{c}+\delta_{x} p \\
\rho v u_{c}+\delta_{y} p \\
\rho w u_{c}+\delta_{z} p \\
\left(E_{t}+p\right) u_{c}
\end{array}\right)
$$

where $J$ is the jacobian of the coordinate transformation and the contravariant velocities, $u_{c}$, are defined in terms of the metric coefficients as

$$
u_{c}=\delta_{x} u+\delta_{y} v+\delta_{z} w
$$

(The viscous fluxes are omitted here for brevity, and can be found in many References, such as Reference 19.) Following the procedure outlined in Ref. 10 and augmented with results shown in References 20 and 21 , the hybrid LU-SGS scheme can be derived from the following. A prototype implicit scheme for solving (1) in delta form may be written as

$$
\left[I+\Delta t\left(D_{\xi} A+D_{\eta} B+D_{\zeta} C\right)\right] \Delta q=-\Delta t R
$$

where $D_{\xi}, D_{\eta}$ and $D_{\zeta}$ are difference operators that approximate $\partial_{\xi}, \partial_{\eta}$ and $\partial_{\zeta}$ and $A, B$ and $C$ are the flux jacobians

$$
A=\frac{\partial E}{\partial q}, B=\frac{\partial F}{\partial q}, C=\frac{\partial G}{\partial q}
$$

The PARC codes calculate the residual, $R$ in (4), using central differences with added artificial dissipation in the manner due to Jameson (Ref. 23). If the flux jacobians in (4) are approximated so that the resulting jacobians can be split into "+" and "-" matrices, where the eigenvalues of the ${ }^{n}+{ }^{n}$ matrices are nonnegative and the eigenvalues of the "-" matrices are non-positive, and then differenced according to their sign, (4) may be written as

$$
\begin{gathered}
{\left[I+\Delta t\left(\nabla_{\xi} A^{+}+\Delta_{\xi} A^{-}+\nabla_{\eta} B^{+}+\Delta_{\eta} B^{-}\right.\right.} \\
\left.\left.+\nabla_{\zeta} C^{+}+\Delta_{\zeta} C^{-}\right)\right]=-\Delta t R
\end{gathered}
$$

The choice of conditioning used to form the approximated jacobians is very important, and following the construction used in Reference 21 , the approximated jacobians are formed as

$$
A^{ \pm}=\frac{A \pm r_{A} I}{2}, B^{ \pm}=\frac{B \pm r_{B} I}{2}, C^{ \pm}=\frac{C \pm r_{C} I}{2}
$$

where

$$
r_{A, B, C}=\beta \max \left\{\left|\lambda_{A, B, C}\right|\right)
$$

where $\beta \geq 1.0$ and $\lambda_{A, B, C}$ are the eigenvalues of the flux jacobians. Therefore, using the conditioning in (7), the unfactored scheme may be written as

$$
\begin{gathered}
{\left[I\left(1+\Delta t\left(r_{A}+r_{B}+r_{C}\right)\right)+\Delta t\left(A_{j+1}^{-}+B_{k+1}^{-}+C_{l+1}^{-}\right.\right.} \\
\left.\left.-A_{j-1}^{+}-B_{k-1}^{+}-C_{l-1}^{+}\right)\right] \Delta q=-\Delta t R
\end{gathered}
$$

where $(j, k, l)$ refers to the grid point index. (When an index is omitted it is assumed to be at $\mathrm{j}, \mathrm{k}$ or $\mathrm{l}$ ). The hybrid LU-SGS scheme is then found by factoring (9) into $L, D$ and $U$ operators so that

$$
\begin{gathered}
L D^{-1} U \Delta q=-\Delta t R \\
L=I\left[1+\Delta t\left(r_{A}+r_{B}+r_{C}\right)\right] \\
-\Delta t\left(A_{j-1}^{+}+B_{k-1}^{+}+C_{l-1}^{+}\right) \\
D^{-1}=I\left[1+\Delta t\left(r_{A}+r_{B}+r_{C}\right)\right] \\
U=I\left[1+\Delta t\left(r_{A}+r_{B}+r_{C}\right)\right] \\
+\Delta t\left(A_{j+1}^{-}+B_{k+1}^{-}+C_{l+1}^{-}\right)
\end{gathered}
$$

All of the calculations performed here are only concerned with obtaining the steady state flowfield, so an approximate Newton iteration formulation is formed by dividing (10) by $\Delta t$ and taking the limit as $\Delta t$ approaches infinity, resulting in

$$
\begin{gathered}
L U \Delta q=-\left(r_{A}+r_{B}+r_{C}\right) R \\
L=\left[I\left(r_{A}+r_{B}+r_{C}\right)-A_{j-1}^{+}-B_{k-1}^{+}-C_{l-1}^{+}\right] \\
U=\left[I\left(r_{A}+r_{B}+r_{C}\right)+A_{j+1}^{-}+B_{k+1}^{-}+C_{l+1}^{-}\right]
\end{gathered}
$$

The solution of (14) is fully vectorizable on planes where $\mathbf{j}+\mathbf{k}+\mathbf{l}=$ constant and is efficient since it requires only scalar diagonal inversions. The approach is to first solve for an intermediate vector using (15) by sweeping in the direction of planes of increasing $j+k+l$ and then solve for $\Delta q$ using (16) by sweeping in the opposite direction. The efficiency in solving the chemical transport equations fully coupled to the Navier-Stokes equations has been demonstrated in References 10,11 and 12 by using the LU-SGS scheme. The only modifications to the implicit operator are to account for the 
larger flux jacobians and the inclusion of the species production/destruction terms implicitly in the first operator, (15). Another advantage of this scheme is that when the approximate Newton iteration form is used, no choice of time step or Courant number is needed. This is very useful since for most problems it is not known a priori what time step or Courant number is needed to gain sufficient convergence.

\section{LU-SGS and DBW Comparison}

Since the present work has replaced the original diagonalized Beam-Warming scheme (hereafter referred to as $\mathrm{DBW}$ ) with this algorithm it was deemed prudent to make comparisons regarding the relative efficiencies of the two schemes. A limited study was made for inviscid $2 D$ flows, and is summarized in the following sections. In all the comparisons, only the implicit operator was changed; the residual calculations were performed in the same manner with the same coefficients of artificial dissipation for both schemes. All calculations were performed on a CRAY-2 using the cft77 compiler.

\section{III.a: $M_{\infty}=3$ Blunt Body}

The inviscid flow over the windward portion of a right circular cylinder at a free stream Mach number of 3 was calculated using the LU-SGS and DBW schemes. A $101 \times 101$ evenly spaced grid was used, resulting in approximately 62 percent of the grid points being in the subsonic portion of the flow. Figure 1 shows the computed flowfield Mach number contours which clearly indicates the curved bow shock and the expansion along the body surface from the stagnation streamline. For the DBW calculations a spatially varying time step was used by holding the Courant number (CN) constant at all the grid points while $\beta$ in (8) was linearly varied from it's maximum, $\beta_{\max }$, to 1.0 over the first 500 iterations for the LU-SGS calculations. Figure 2 compares the convergence histories of the LU-SGS and DBW calculations on a work basis, where one unit of work is equal to the cpu time for one iteration of the DBW scheme, for a range of Courant numbers and $\beta_{\max }$ values. The Figure clearly shows the increasing rates of convergence with increasing CN for the DBW scheme and the relative insensitivity to $\beta_{m a x}$ of the LU-SGS scheme. At CN's higher than 2, the oscillations present in the beginning stages of the iteration persisted over a longer range, retarding the convergence to less than that for the $\mathrm{CN}=2$ calculation. This Figure shows that the two schemes converged at roughly the same rates, but only when the DBW was run at a proper Courant number.
For this case, the LU-SGS scheme was roughly 10 percent faster in time per iteration than the DBW scheme.

\section{III.b: Transonic Nozzle}

A simplified transonic nozzle flowfield was calculated using the LU-SGS and DBW schemes. The geometry was constructed by matching two cubic polynomials at the throat region with the inlet and exit heights determined from 1-D theory to provide an inlet Mach number of 0.2 and an exit Mach number of 3.0. The total temperature and pressure were held constant at the inflow boundary and the static pressure was held constant at the exit. A 101 X 51 evenly spaced grid was used for this calculation. Figure 3 shows the computed Mach number contours and geometry of the nozzle, showing that the flow accelerates smoothly through the throat and then rapidly becomes supersonic, where an excessive ammount of turning causes a weak shock near the upper nozzle lip. As in the previous case, spatially varying time stepping was used for the $D B W$ scheme by holding the Courant number constant at all the grid points, while $\beta$ was varied linearly from a maximum to 1.0 over 500 iterations. Figure 4 compares the LU-SGS and DBW calculations for a range of Courant numbers (CN) and values of $\beta_{\max }$. As before, increasing the CN improved the convergence rate of the DBW scheme, but a limit was reached where beyond a $\mathrm{CN}=3.6$, the $\mathrm{DBW}$ scheme diverged. Also, the LU-SGS was fairly insensitive to the $\beta$ variation. For this case the LU-SGS was approximately 20 percent faster in time per iteration than the DBW scheme. The difference in efficiency when compared to the first case is attributable to the difference in the vector lengths (i.e.- grid sizes). Again, both schemes performed similarily, but only when the proper Courant number was used for the DBW scheme.

\section{III.c: $M_{\infty}=2.5$ Supersonic Inlet}

The inviscid flow through a generic supersonic inlet at a freestream Mach number of 2.5 was calculated using the two schemes. The computational grid was uniformly spaced in both coordinate directions for this 200 (axially) by 75 (normally) grid point calculation. Figure 5 shows the computed pressure contours in the inlet, indicating the passing of the ramp shock out of the domain and the cowl generated shock attenuation and propagation into the inlet, terminating in a large sonic region. The DBW calculation used a spatially varying time step and the LU-SGS calculation used a $\beta$ that varied from 1.5 to 1.0 over the first 100 iterations. The comparison of the $\mathrm{DBW}$ (with a $\mathrm{CN}=5.0$ ) 
and the LU-SGS schemes on a work basis is shown in Figure 6. For this case, the LU-SGS scheme performed approximately 40 percent faster per iteration than the DBW scheme. The Figure shows that the two schemes converged roughly the same at the early stages of the iteration, but the LU-SGS scheme converged faster in the later stages.

\section{III.d: $M_{\infty}=3.5$ Supersonic Inlet}

This case is identical to the previous case, except the freestream Mach number was increased to $M=3.5$. The DBW scheme uses the same time stepping and Courant number as before, and the LU-SGS uses the same $\beta$ variation. Figure 7 shows the computed pressure contours in the inlet, showing that the ramp shock does not get ingested and the cowl shock is strong enough to to travel throughout the inlet. Figure $8 \mathrm{com}$ pares the two schemes convergence behavior for this case on a work basis. The Figure shows, as in the previous case, that the two schemes converge similarily in the early stages of the iterations while the LU-SGS scheme converges faster in the latter stages. As in the previous case, the LU-SGS scheme performed approximately 40 percent faster per iteration than the DBW scheme.

\section{III.e: Summary of Convergence Study}

The results of this study comparing the convergence rates and efficiencies of the LU-SGS and DBW schemes show that the LU-SGS scheme is slightly more efficient on a work basis than the DBW scheme. Since the LU-SGS vectorization is performed on planes in $3 \mathrm{D}$ (lines in $2 D$ ) where $j+k+l=$ constant the efficiency of the scheme is dependent upon the grid dimension since the vector lengths will be short in the plane corners and longer in the center of the planes. Although the convergence rates were nearly the same on an iteration basis, the $\mathrm{DBW}$ scheme required more user effort to find the best time step or Courant number, while for the cases considered here, the LU-SGS was fairly insensitive to "tuning" of the implicit dissipation. Since one often does not know what the "best" time stepping (or Courant number) is needed, this study indicates that the LU-SGS for approximate Newton iteration scheme may be easier to use.

\section{IV: Corner and Gap Seal Flow Field Study}

Effort is currently underway to investigate the heat transfer and aerodynamic loads in and around a scramjet module gap seal configuration. The seal system must prevent high temperature and pressure gases from leaking through the gaps between the articulating engine panel walls and stationary splitter panels (Ref. 13). This system is crucial to the design of hypersonic airbreathing propulsion systems and presents a significant challenge to structural designers to build a viable seal system. To assist the design effort, the present research complements the structural analysis underway in an attempt to better understand the fluid dynamic and heat transfer phenomena in and around the gap seal system.

\section{IV.a: Hypersonic Corner Validation}

To obtain some level of confidence in the modelling of the gap seal flow it is necessary to validate the 3D Navier-Stokes solver with experimental data taken from a class of flows similar to the gap seal flow. Figure 9 shows the simplified geometry of a typical gap seal, showing that the geometry is similar to two mutually perpendicular flat plates (corner) aligned with the freestream flow with "groove" situated at the plate intersections. Due to this similiarity, the 3D code has been validated against the experimental data of hypersonic corner flow taken by Cresci (Ref. 14). These tests were conducted at a Mach number of 11.8 over a range of Reynolds numbers of $0.15 \times 10^{6}$ to $0.5 \times 10^{6}$ per foot. The data presented in Ref. 14 is axial pressures and heat transfer rates plotted against the hypersonic interaction parameter

$$
\chi=\frac{c^{\frac{1}{2}} M_{\infty}^{3}}{\operatorname{Re}_{x}^{\frac{1}{2}}}
$$

for different lateral locations measured from the corner. In addition, surface pressures, heat transfer rates and skin friction data are available (Ref. 15) as a function of lateral distance from the corner at various axial locations. Due to the simple geometry of this case, other researchers have successfuly used this data to validate space marching (Ref. 16) and Navier-Stokes (Ref. 17) calculations. This data not only tests the prediction of the mutual interaction of the two plate boundary layers upon each other but also the self induced leading edge pressures caused by the boundary layer growth close to the leading edges. To test the predictions of the 2D self induced leading edge shocks and heat transfer rates, the $2 D$ and $3 D$ codes were run and compared with the experimental data of Ref. 14 (taken at a lateral distance sufficiently far from the corner to ensure two dimensionality) and with the theoretical pressures deduced by Bertram (Ref. 18). The freestream conditions 
were set at the lower Reynolds number and stagnation condtions from the experiment $\left(M_{\infty}=11.8, \mathrm{Re}=0.15 \mathrm{X}\right.$ $10^{8} /$ foot and $T_{0}=1700$ Rankine) while the wall temperature was held fixed at $\frac{T_{\infty}}{T_{\infty}}=8.6543$. Figures 10 and 11 show the predicted and measured surface pressures and heat transfer rates versus $\chi$ with the experimental and theoretical data (Ref. 18). A $36 \times 41$ grid was used with the grid clustered near the wall using a Robert's transformation (Ref. 19) with $\beta=1.01$ and axially near the leading edge with $\beta=1.1$. Five grid points were extended ahead of the leading edge to aid in correctly capturing the leading edge shock strength. The 3D code used a similar grid with $11 \mathrm{XY}$ planes and symettric boundary condtions applied at the lateral boundaries. As seen in the Figure, the wall pressures were slightly over predicted while the heat transfer rates were predicted very well.

The results from the $2 D$ study were then used to guide the 3D corner flow computations. Two grid sizes were used in the study $(36 \times 41 \times 41$ and $36 \times 61 \times$ 61) for plate lenghts of 8 inches and plate widths of 2.5 inches at a Reynolds number of $0.15 \times 10^{6}$. The grids were clustered normally using the Robert's transformation in both planes with $\beta=1.01$ and axially near the leading edge using $\beta=1.1$ with five points extended ahead of the leading edge. The convergence histories for the both grids using the LU-SGS scheme are shown along with a coarse grid calculation using the DBW scheme in Figure 12. The DBW calculation was made at a constant Courant number of 2.5 , and contrary to the $2 \mathrm{D}$ inviscid results, the LU-SGS was only 3 percent faster in time per iteration than the DBW scheme. This Figure shows that for this case the LU-SGS computations seemed to damp the initial transients more quickly than the DBW and that the convergence rate at the latter stages of the iterations was faster for the LU-SGS scheme. At the inflow plane the flux variables were held constant at their freestream values, constant temperature no-slip wall conditions were applied on the plates and grid extrapolation of the primitive variables was used at the outflow and lateral planes. Figures 13.a,b,c and $d$ show the predicted and measured wall heat transfer rates measured laterally from the corner at different axial locations. Figures $14 . a$ through e compare the predicted and measured wall pressures at different lateral locations plotted versus the hypersonic interaction parameter. Figure 15 shows spanwise surface pressure comparisons while Figure 16 shows the spanwise skin friction coefficients. Figures $13 \mathrm{a}, \mathrm{b}$ and $d$ show remarkably good comparisons between the predicted and measured heat transfer rates, peak levels and peak locations, while Figure 13.c shows the correctly predicted level but incorrectly predicted peak location. The pressure comparisons show good agreement near the corner
(Figures 14.a,b and c) but get worse at farther distances from the corner (Figures 14.d and e). The experimental data indicates that the plate pressure returns to the $2 \mathrm{D}$ pressure at a distance of approximately 1.5 inches from the corner at $\chi=5.0$, but the present calculations only show a very gradual lateral reduction in pressure from the peak corner values. This behavior is similar to the computed results in Ref. 17 in that the near corner pressures are computed well, but the spanwise pressure relief is underpredicted.

\section{IV.b: Gap Seal and Corner Comparisons}

In an attempt to characterize the gap seal flow, comparisons were made between corner and gap seal flows at similar Reynolds and Mach numbers. Computations were made using the LU-SGS scheme for a Mach number of 10 at an altitude of 100,000 feet of the flow fields through a corner and a gap seal configuration. At this altitude the freestream pressure and temperature are 23.085 psf and 418.79 Rankine yielding a Reynolds number per foot of $1.0 \times 10^{6}$. The length of the plates and gap seal configurations were taken to be 5 inches with widths of 1 inch, resulting in a Reynolds number based on plate length of 420,000 . The gap seal "groove" (shown in Figure 9) was taken to be $1 / 8$ by $1 / 8$ inches and is considered to run along the entire length of the plates. The wall temperatures were held constant at $1200 \mathrm{Fahrenheit}$. The corner flow computation used a $36 \times 51 \times 51$ grid with the same clustering as the previous corner case with 5 points extended ahead of the leading edge to help capture the leading edge shock strengths. The gap seal computation used a rectangular grid with clustering centered along the gap seal faces and toward the plate walls as well as axial clustering near the leading edge with 5 leading edge extension points. This computation used a base grid of $36 \times 61 \times 61$ of which approximately 75 percent of the grid points were active in the calculation. Figure 17 compares the convergence rates of the corner and gap seal computations using the LU-SGS scheme, showing that both cases converged monotonically and rapidly towards steady state.

Figures 18.a,b and compare the wall heat transfer coefficients along the gap seal floor and lateral wall with the corner only heat transfer rates for different axial locations. Examination of the Figures shows the peak heat transfer to the gap seal floor is reduced by approximately 4 times while the lateral wall heat transfer levels are approximately the same as the corner only levels. This reduction in heat transfer in the gap seal is due the the gap region filling up with low momentum and energy fluid caused by the viscous effects in 
the gap. Figure 19 shows axial velocity contours near the end of the seal configuration in a plane perpendicular to the free stream velocity vector. This Figure shows the gap region filled with low velocity fluid and the existence of a vortical flow situated a short distance laterally from the gap seal. This vortical flow is caused by the rapid expelling of the flow from the gap seal to the outer lateral plate. Future computations will investigate whether this vortex will act as a barrier to keep the energetic outer flow from entering the gap seal region.

\section{V: Conclusions}

A hybrid LU-SGS scheme has been successfully implemented in the PARC2D/3D Navier-Stokes solvers and limited comparisons to the original algorithm in the code (diagonalized Beam-Warming ADI) have been made. The convergence comparisons indicated that the hybrid LU-SGS scheme for approximate Newton iteration is slightly more efficient on a work basis than the DBW scheme and requires less user effort to achieve an acceptable convergence rate. This is most likely due to a combination of the inherent implicit dissipation of the LU-SGS scheme and the approximate Newton iteration formulation, so that no time step specification is required. The scheme was demonstrated by validating the code against a $M_{\infty}=11.8$ corner flow experiment and has been used to investigate the similarities between gap seal and corner flow geometries. The validation effort indicated that the code predicted the corner case heat transfer rates, peaks and peak locations very well while it predicted the wall pressures very well near the corner but poorly away from the corner. The gap seal/corner flow field comparisons indicated that the heat transfer rates to the floor of the gap seal were signifigantly reduced when compared to the corresponding corner only flow and that the lateral heat transfer peaks were displaced laterally by approximately the gap width distance. Future work will investigate the gap seal/corner flows in the limit of large and small gap aspect ratios as well as the inclusion of inert gas "blow by" cooling through the gap seal floor.

\section{References}

(1) Pulliam, T.H., and Steger, J.L. "Implicit FiniteDifference Simulations of Three Dimensional Compressible Flow." AIAA Journal, Vol. 18, No. 2, Feb. 1980, pp. 159-167.

(2) Beam, R. and Warming, R.F. "An Implicit FiniteDifference Algorithm for Hyperbolic Systems in
Conservation-Law Form." Journal of Computational Physics, Vol. 22, No. 1, Sept. 1976, pp. 87-110.

(3) Pulliam, T.H. "Euler and Thin Layer NavierStokes Codes: ARC2D,ARC3D." Notes for Computational Fluid Dynamics User's Workshop, The University of Tennessee Space Institute, Tullahoma, Tennessee, (UTSI Publication E02-4005023-84), March 12-16, 1984, pp. 15.1-15.85.

(4) Cooper, G.K. "The PARC Code: Theory and Usage." AEDC-TR- 87-24, October, 1987.

(5) Jameson, A. and Turkel, E. "Implicit Schemes and LU Decompositions." Mathematics of Computation, Vol. 37, No. 156, 1981, pp. 385-397.

(6) Jameson, A. and Yoon, S., "Lower-Upper Implicit Schemes with Multiple Grids for the Euler Equations." AIAA Journal, Vol. 25, July 1987, pp. 929935.

(7) Jameson, A. and Yoon, S., "Multigrid Solution of the Euler Equations using Implicit Schemes.", AIAA Journal, Vol. 24, Nov. 1986, pp. 1737-1743.

(8) Yoon, S. and Jameson, A., "Lower-Upper Implicit Scheme for High-Speed Inlet Analysis.", AIAA Journal, Vol. 25, Aug. 1987, pp 1052-1053.

(9) Yoon, S. and Jameson, A., "An LU-SSOR Scheme for the Euler and Navier-Stokes Equations.", jAIAA paper 87-0600, January, 1987.

(10) Shuen, J.S. and Yoon, S. "Numerical Study of Chemically Reacting Flows Using an LU Scheme." AIAA paper 88-0436, January, 1988.

(11) Yu, S.T., Tsai, Y.L. and Shuen, J.S. "ThreeDimensional Calculation of Supersonic Reacting Flows using an LU Scheme." AIAA paper 89-0391, January, 1989.

(12) Park, C. and Yoon, S. "Calculation of Real-Gas Effects on Blunt-Body Trim Angles.", AIAA paper 89-0685, Jan. 1989.

(13) Steinetz, B.M., DellaCorte, C. and Sirocky, P. "On the Development of Hypersonic Engine Seals." NASA TP-2854, Dec. 1988.

(14) Cresci, R.J. "Hypersonic Flow Along Two Intersecting Planes.", AFOSR 66-0500, March 1966.

(15) Cresci, R.J., Rubin, S.G., Nardo, C.T. and Lin, T.C. "Hypersonic Interaction along a Rectangular Corner." AIAA Journal, Vol. 7, No. 12, p 2241 2246.

(16) Buggeln, R.C., Kim, Y.N. and McDonald, H. "Computation of Multi-Dimensional Viscous $\mathrm{Su}$ personic Flow.", NASA CR-4021, Oct. 1986.

(17) Li, C.P. "A Numerical Study of the Axial Corner Flow.", AIAA paper 78-1146, July, 1978.

(18) Bertram, M.H. and Blackstock, T.A. "Some Simple Solutions to the Problem of Predicting Bound- 
ary - Layer Self-Induced Pressures.", NASA TND-798, April 1961.

(19) Anderson, D.A., Tannehill, J.C. and Pletcher, R.H. "Computational Fluid Mechanics and Heat Transfer." McGraw-Hill Book Company, 1984.

(20) Yoon, S. and Kwak, D. "Artificial Dissipation Models for Hypersonic Internal Flows." AIAA paper 88-3277, July, 1988 .

(21) Yoon, S. and Kwak, D. "Artificial Dissipation Models for Hypersonic External Flows." AIAA paper 88-3708, July, 1988.

(22) Jameson, A., Schmidt, W. and Turkel, E. "Numerical Solutions of the Euler Equations by Finit Volume Methods Using Runge-Kutta Time-Stepping Schemes." AIAA paper 81-1259, 1981. 


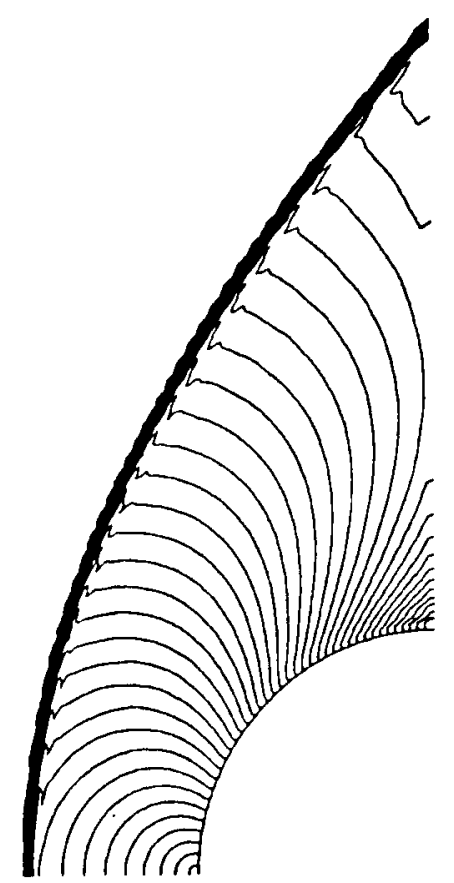

Figure $1 M_{\infty}=3$ Blunt Body: Mach Contours

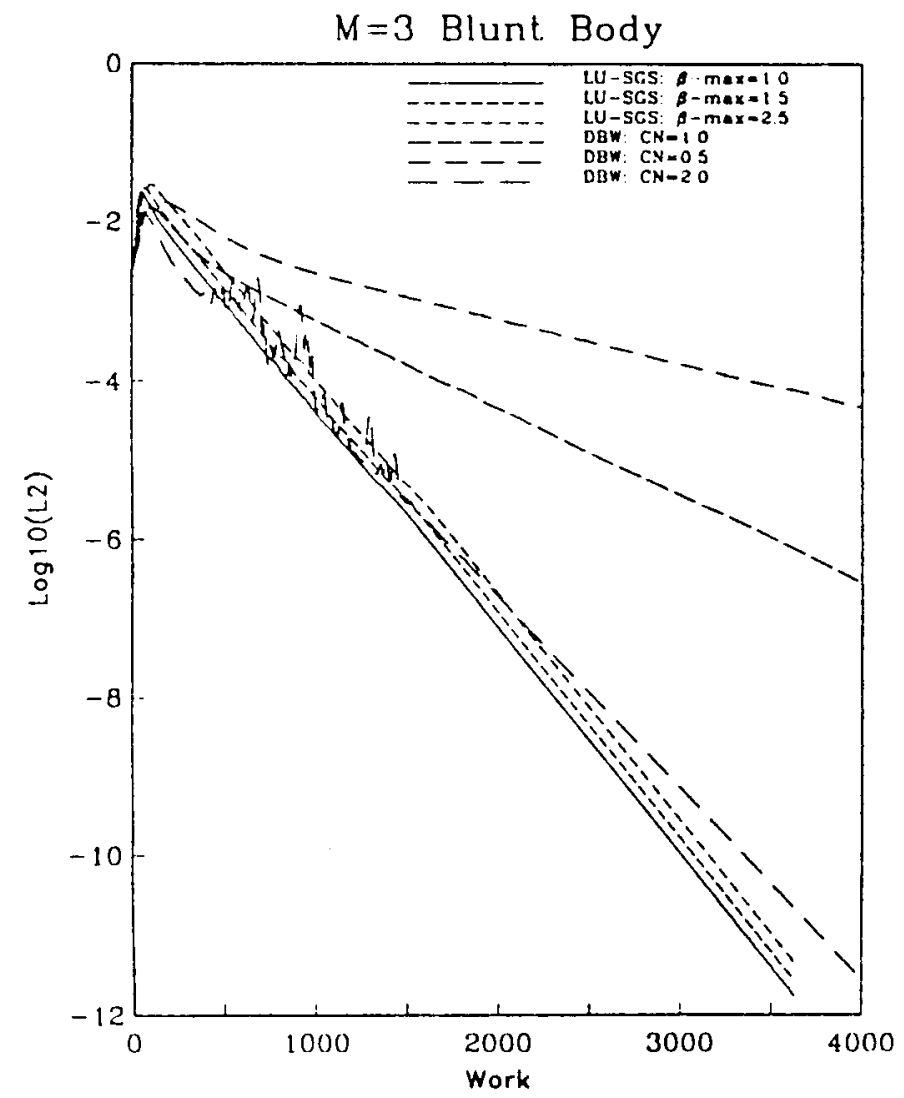

Figure $2 M_{\infty}=3$ Blunt Body: Convergence Comparisons

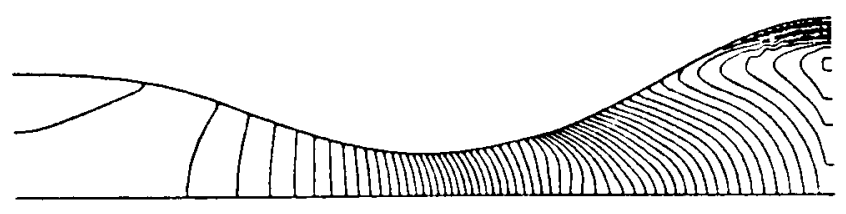

Figure 3 Transonic Nossle Case: Mach Contours

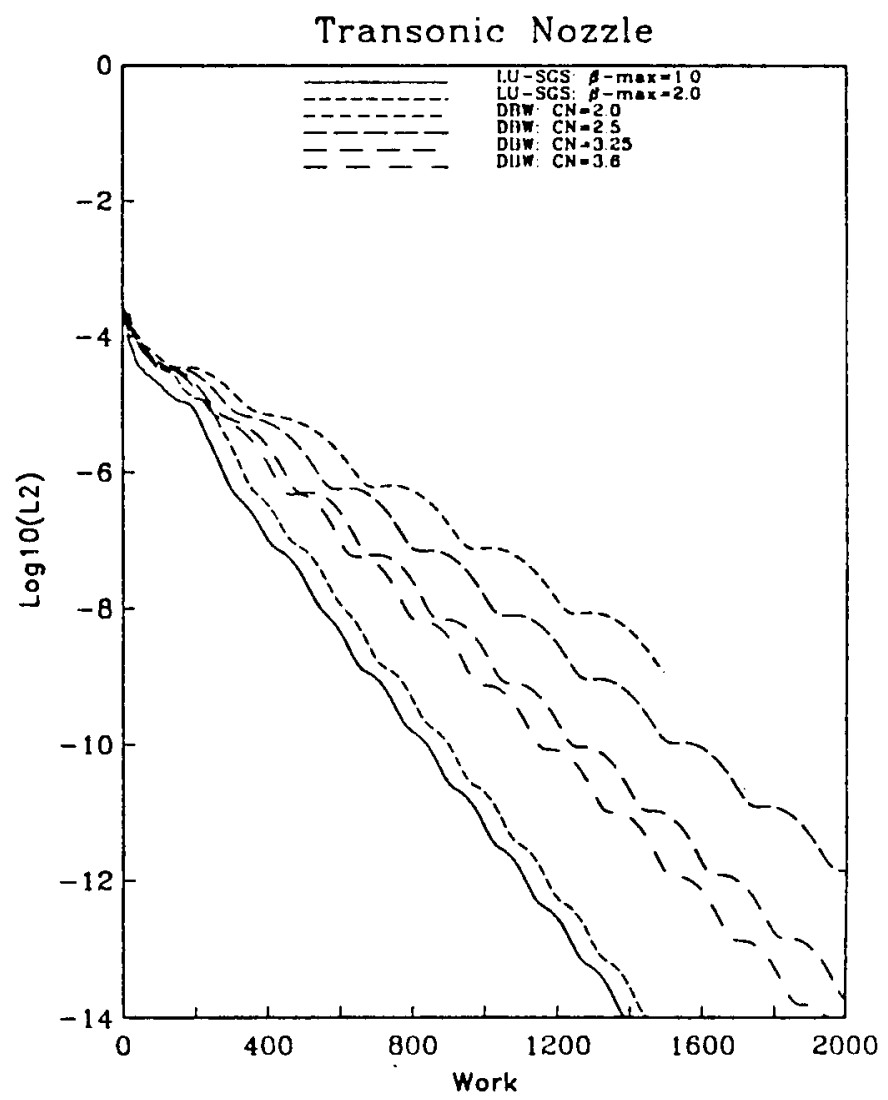

Figure 4 Transonic Nossle Case: Convergence Comparisons 


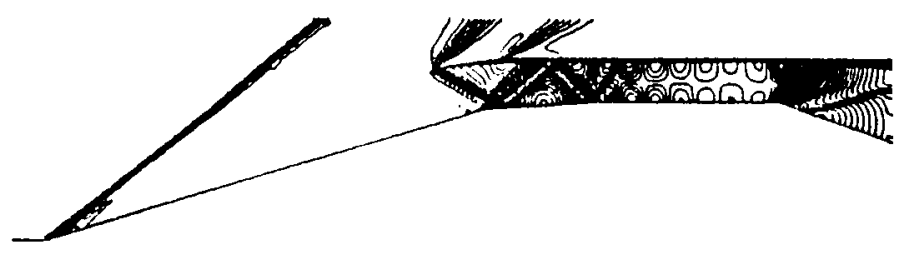

Figure $5 M_{\infty}=2.5$ Supersonic Inlet: Pressure Contours
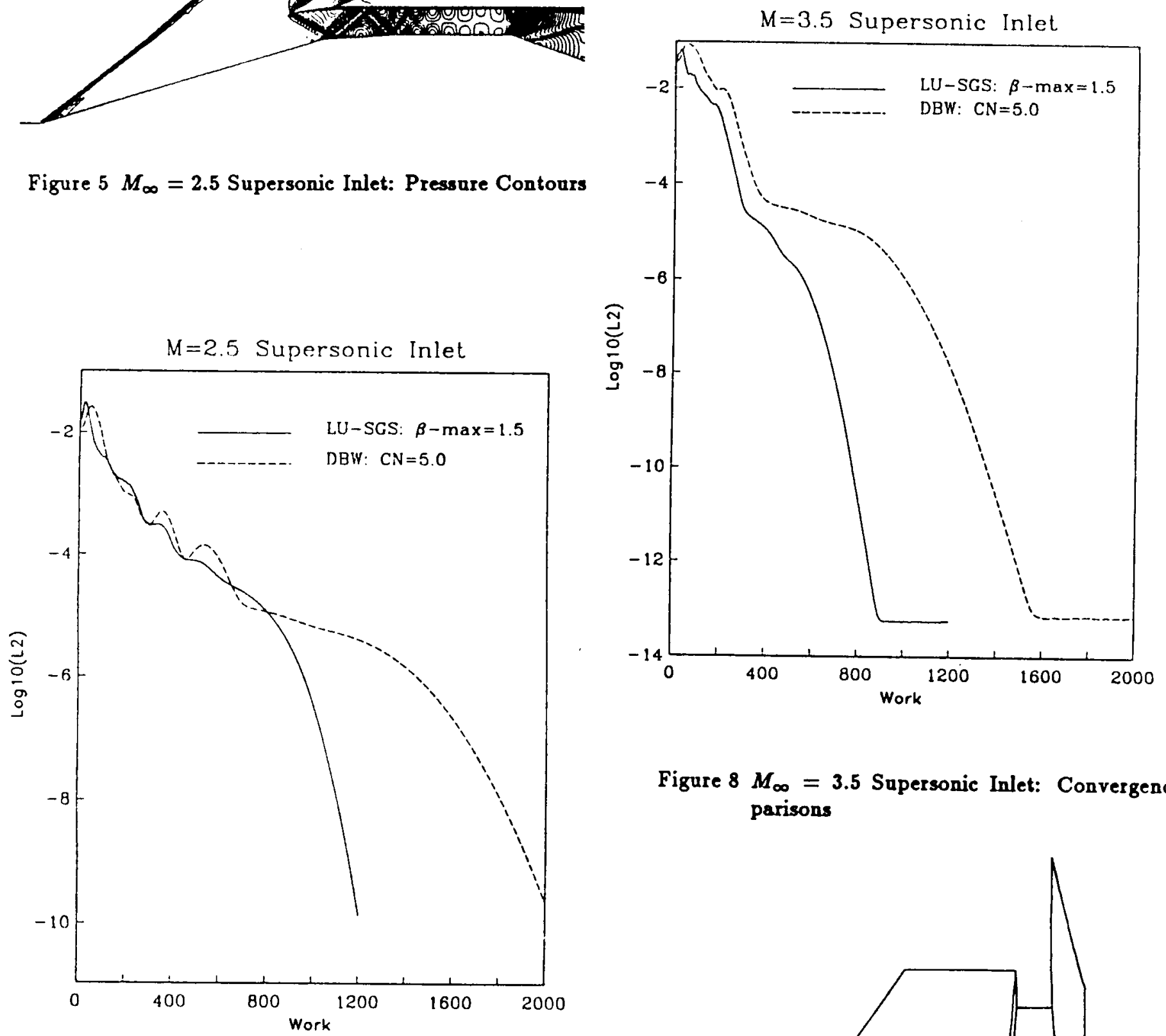

Figure $6 M_{\infty}=2.5$ Supersonic Inlet: Convergence Comparisons

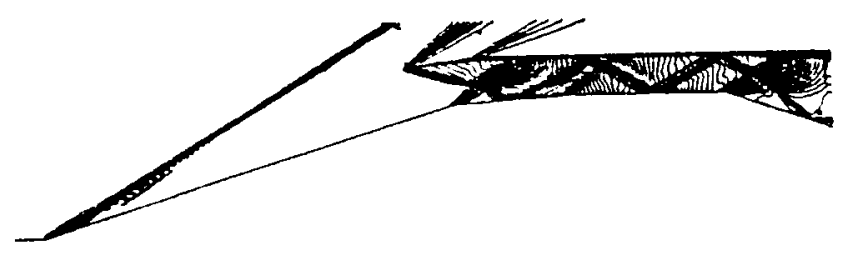

Figure $7 M_{\infty}=3.5$ Supersonic Inlet: Pressure Contours

Figure $8 M_{\infty}=3.5$ Supersonic Inlet: Convergence Comparisons

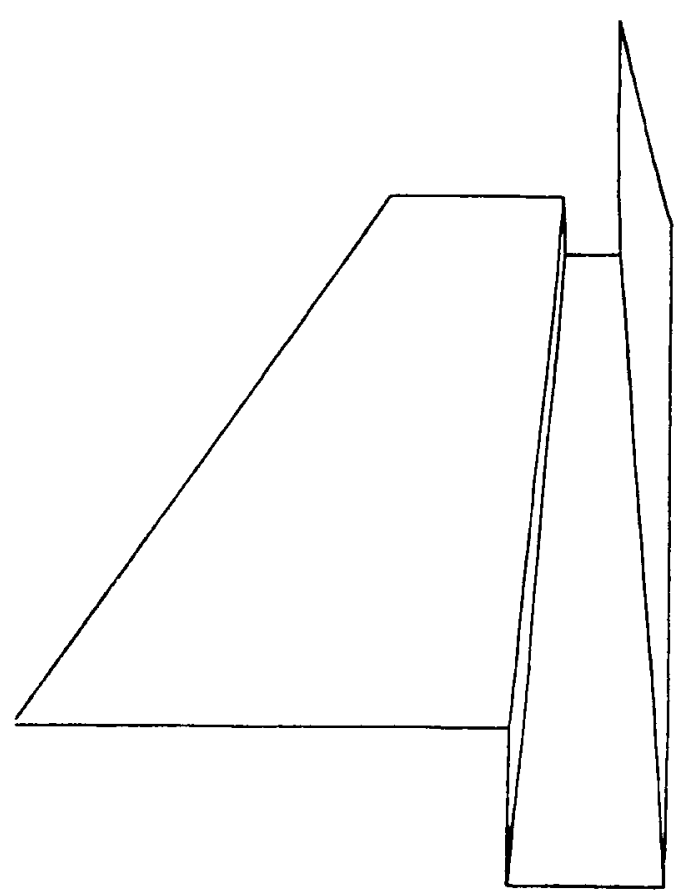

Figure 9 Simplified Gap Seal Geometry 


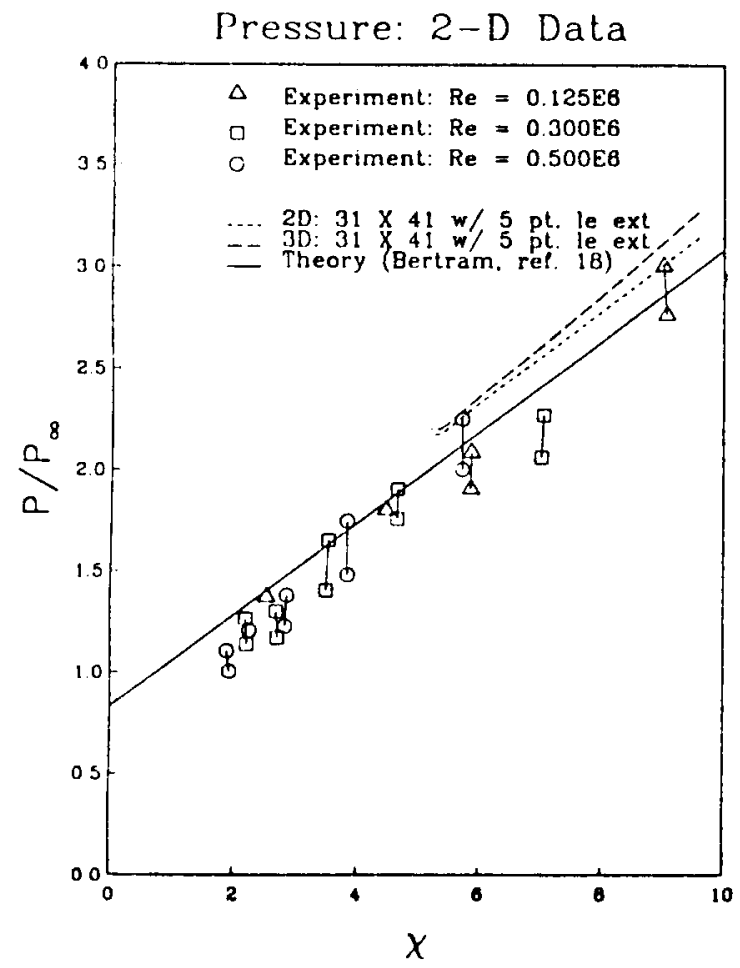

Figure 10 Two-Dimensional Self Induced Leading Edge Plate Pressures

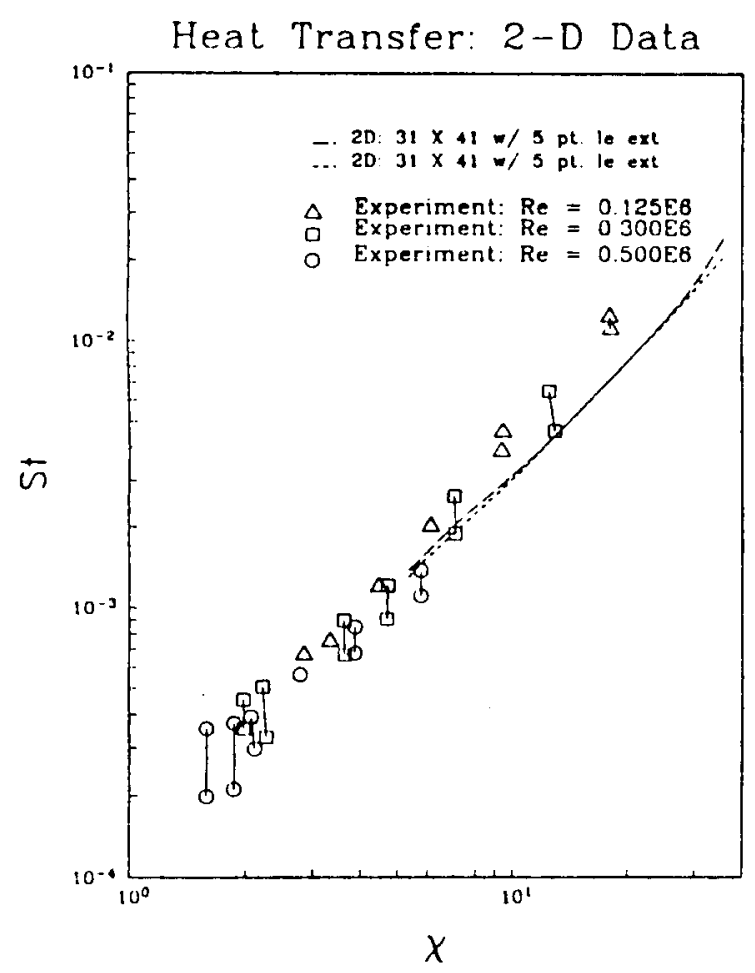

Figure 11 Two-Dimensional Heat Transfer Rates

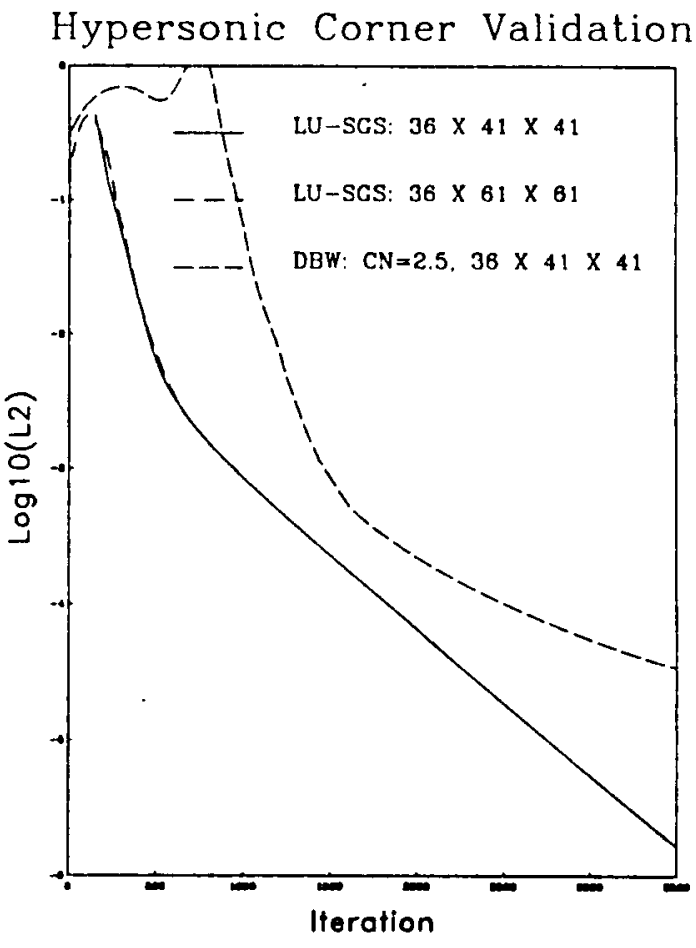

Figure 12 Three-Dimensional Convergence Comparisons

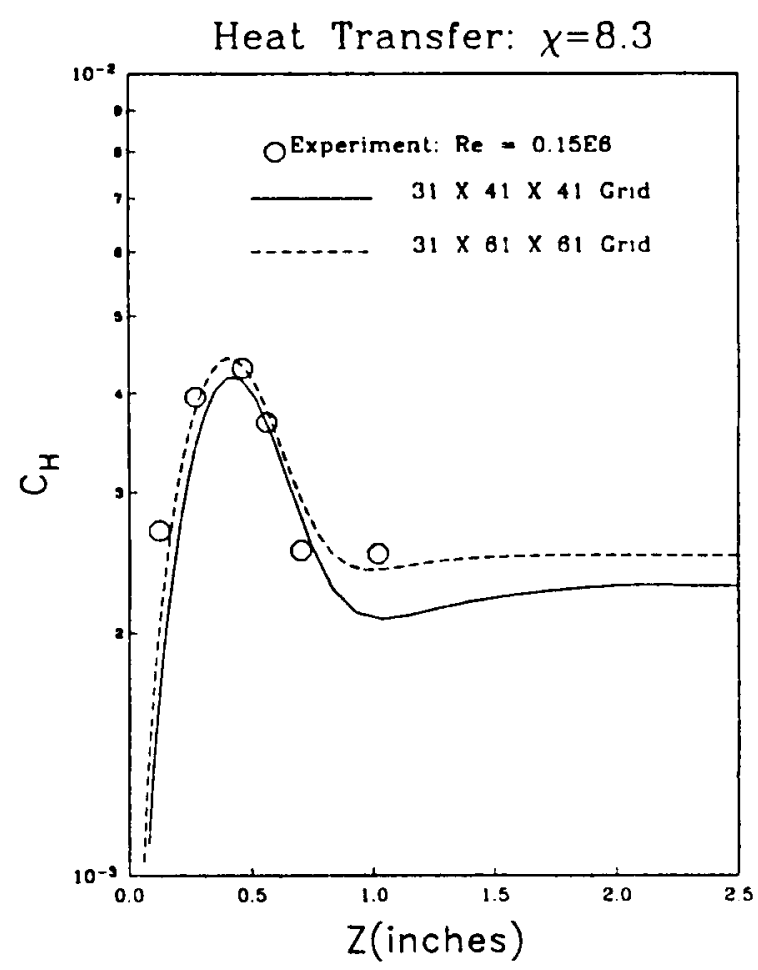

Figare 13 (a)Lateral Variation of Heat Transfer: $\chi=8.3$ 


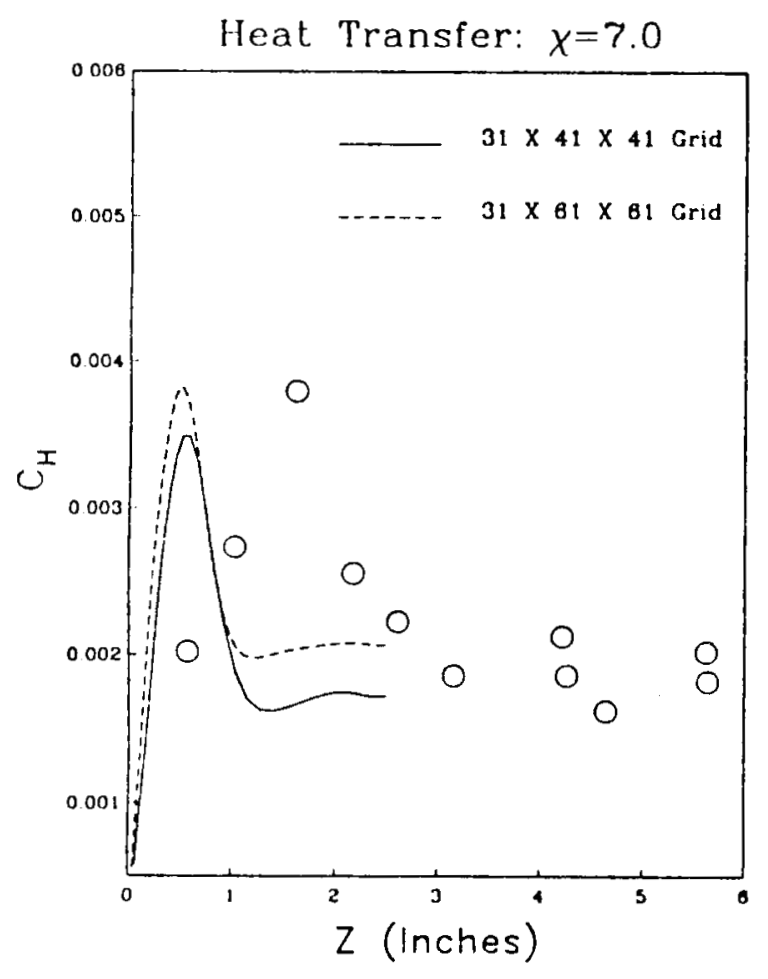

Figure 13 (b)Lateral Variation of Heat Transfer: $\chi=7.0$

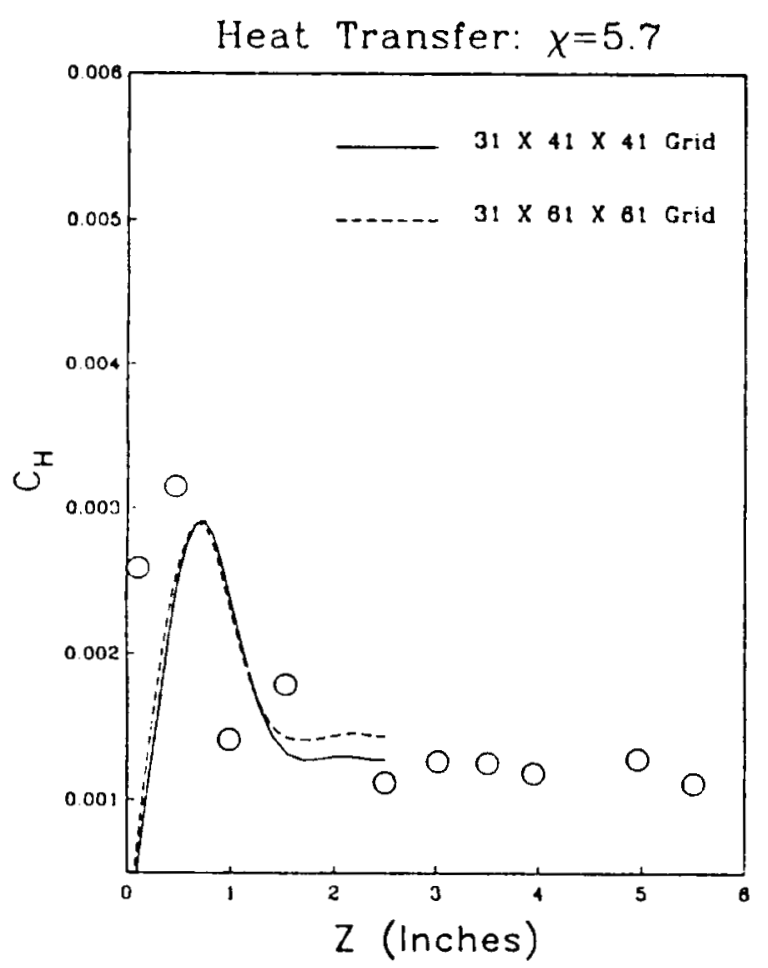

Figure 13 (c)Lateral Variation of Heat Transfer: $\chi=5.7$

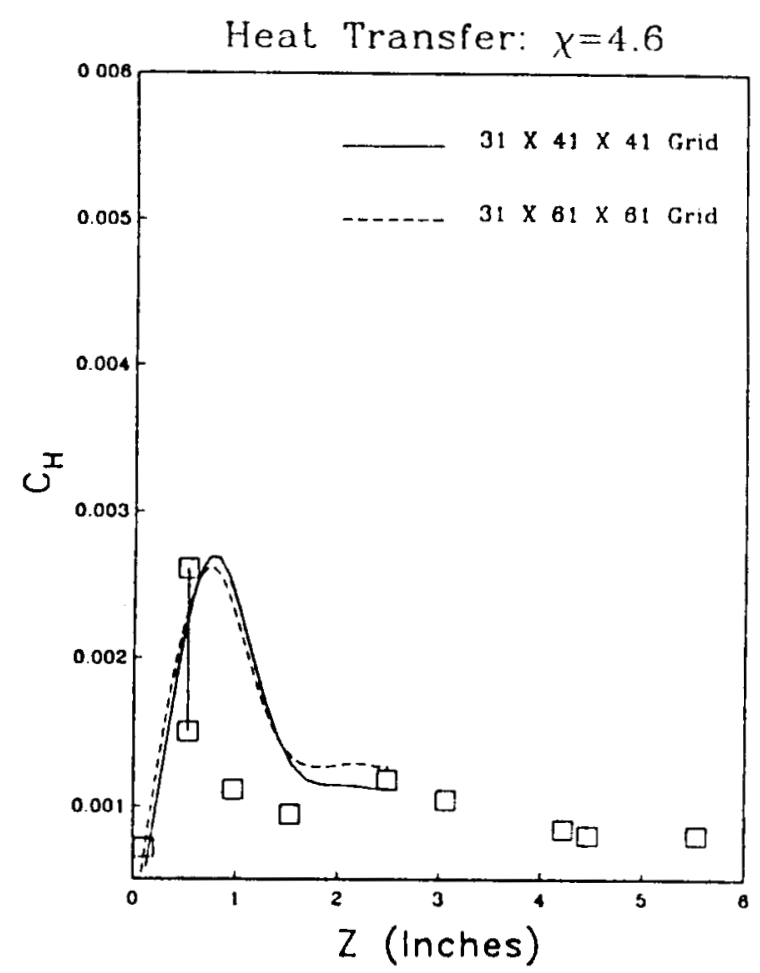

Figure 13 (d)Lateral Variation of Heat Transfer: $\chi=4.6$

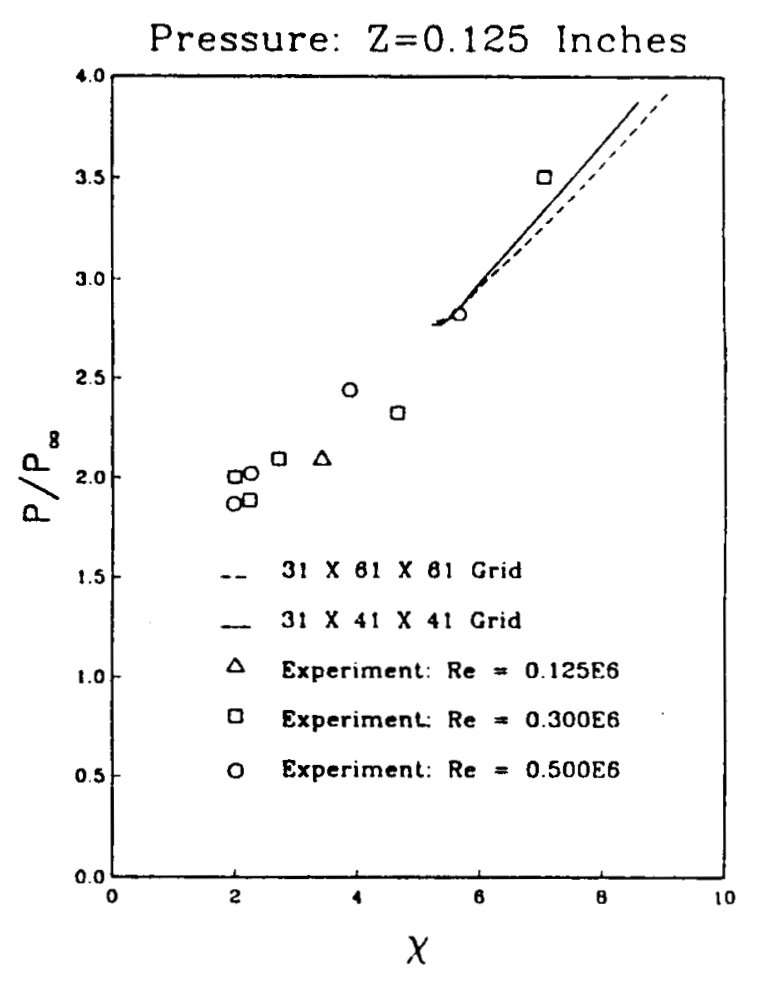


Pressure: $\mathrm{Z}=0.25$ Inches

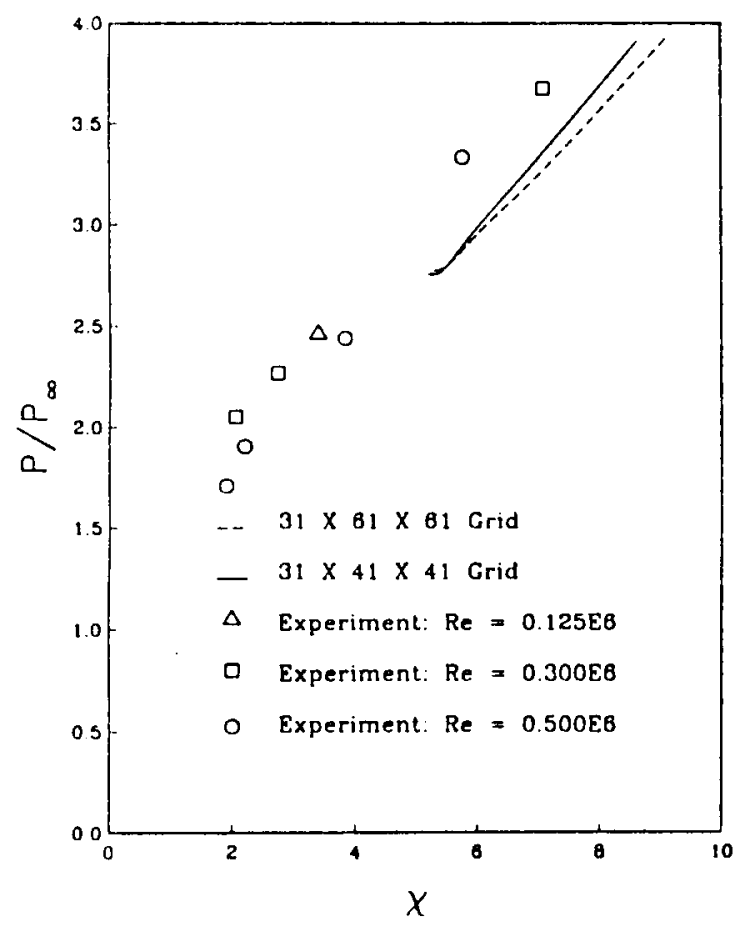

Figure 14 (b)Arial Variation of Wall Pressure: $\mathrm{Z}=0.250$ inches

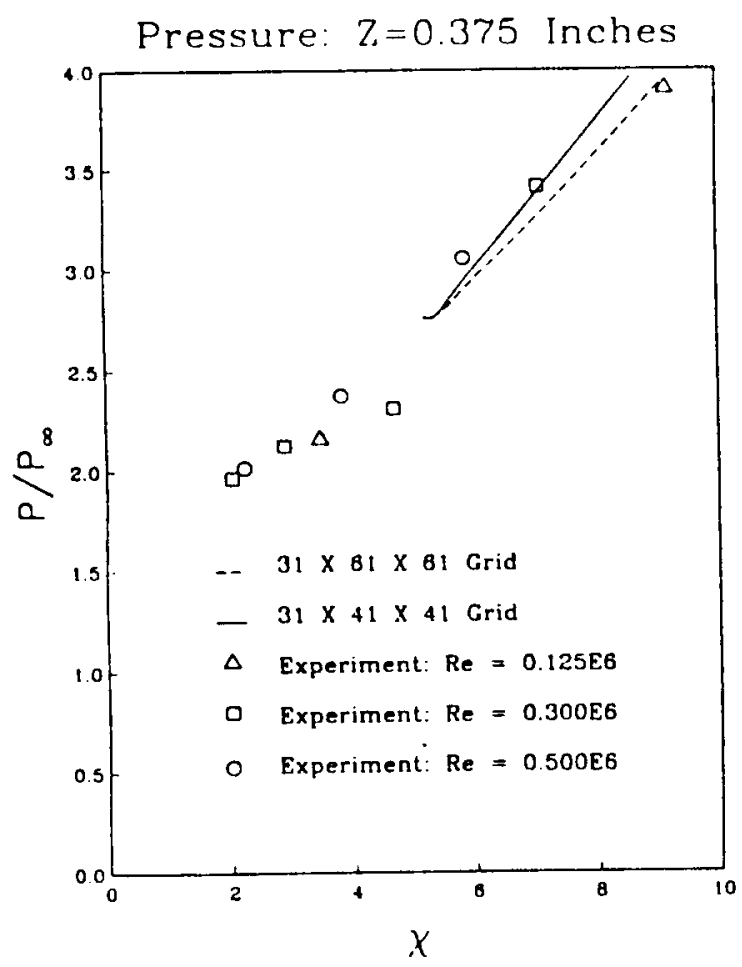

Figure 14 (c)Arial Variation of Wall Pressure: $Z=0.375$ inches

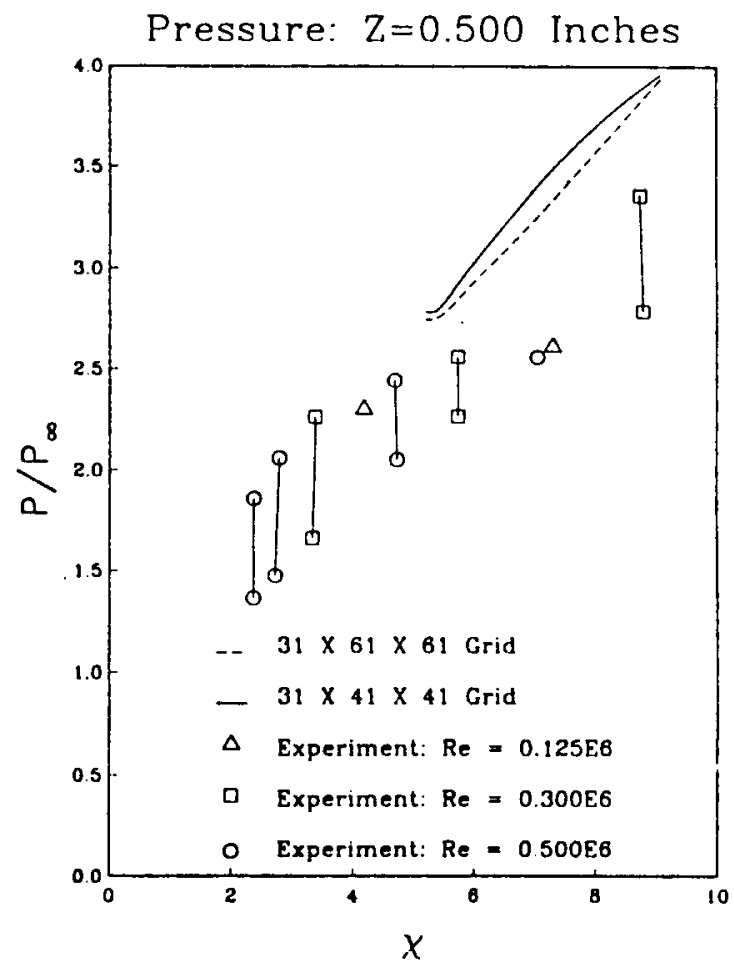

Figure 14 (d)Axial Variation of Wall Pressure: $Z=0.500$ inches

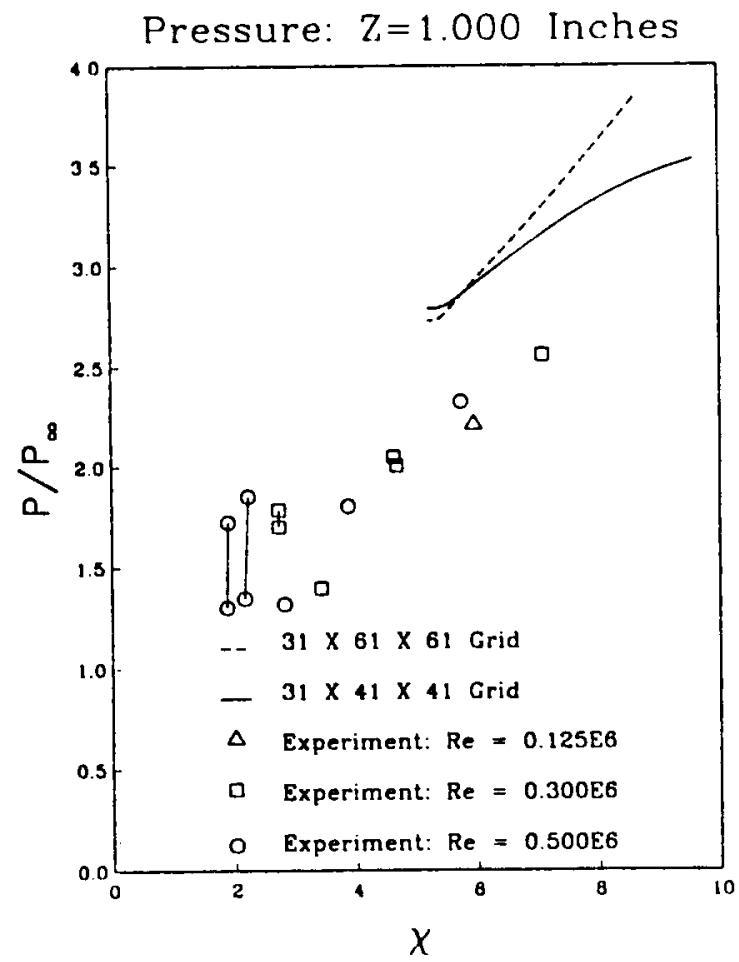

Figure 14 (e)Arial Variation of Wall Pressure: $Z=1.000$ inches 


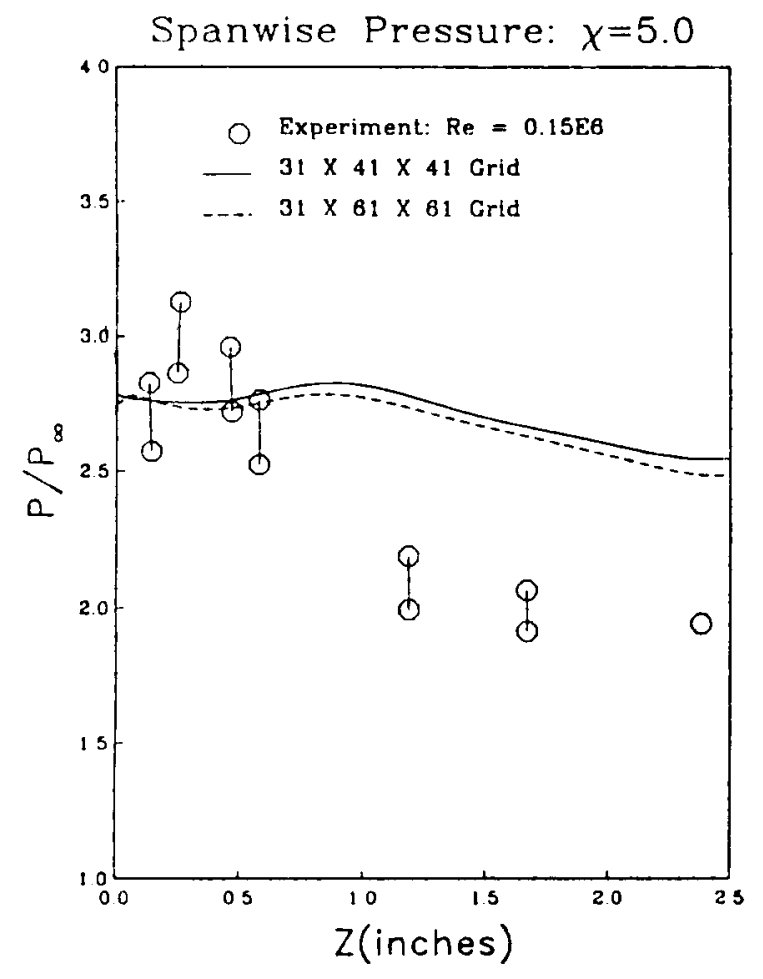

Figure 15 Lateral Pressure Distribution at $\chi=\mathbf{5 . 0}$

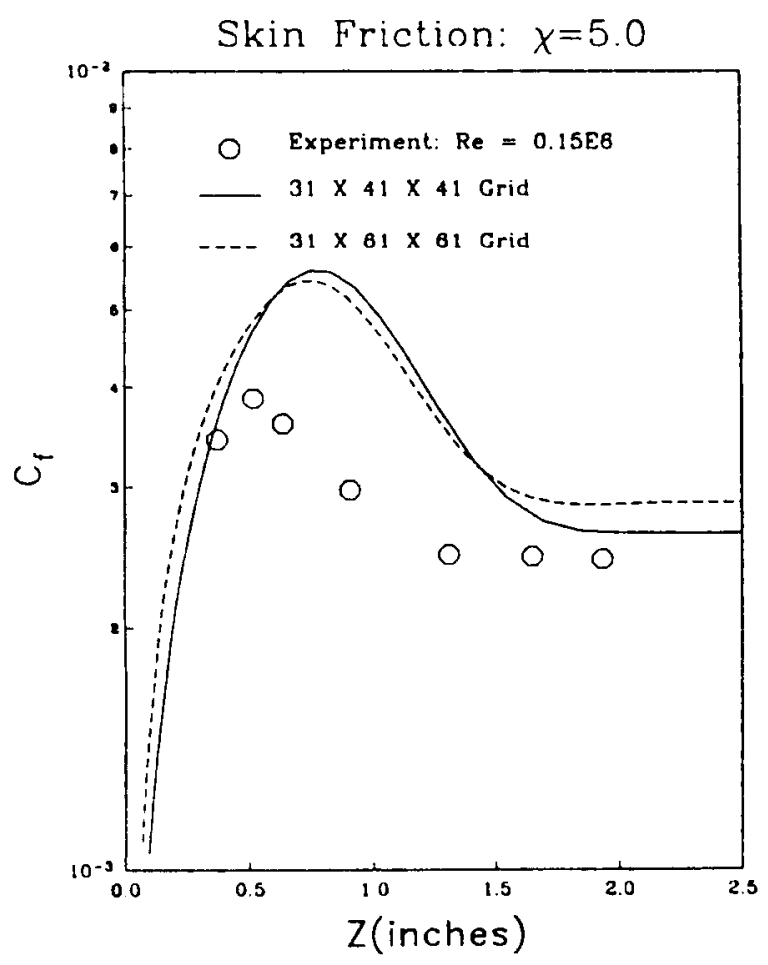

Figure 16 Lateral Skin Friction Distribution at $\chi=\mathbf{5 . 0}$
Hypersonic Corner/Gap Seal

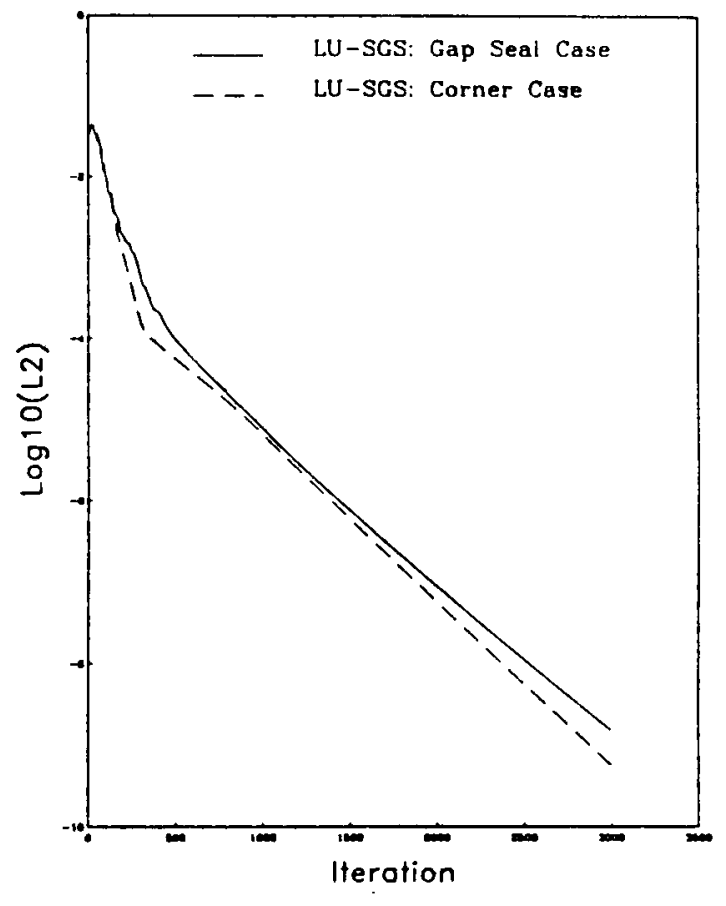

Figure 17 Convergence History: Corner and Gap Seal Comparison

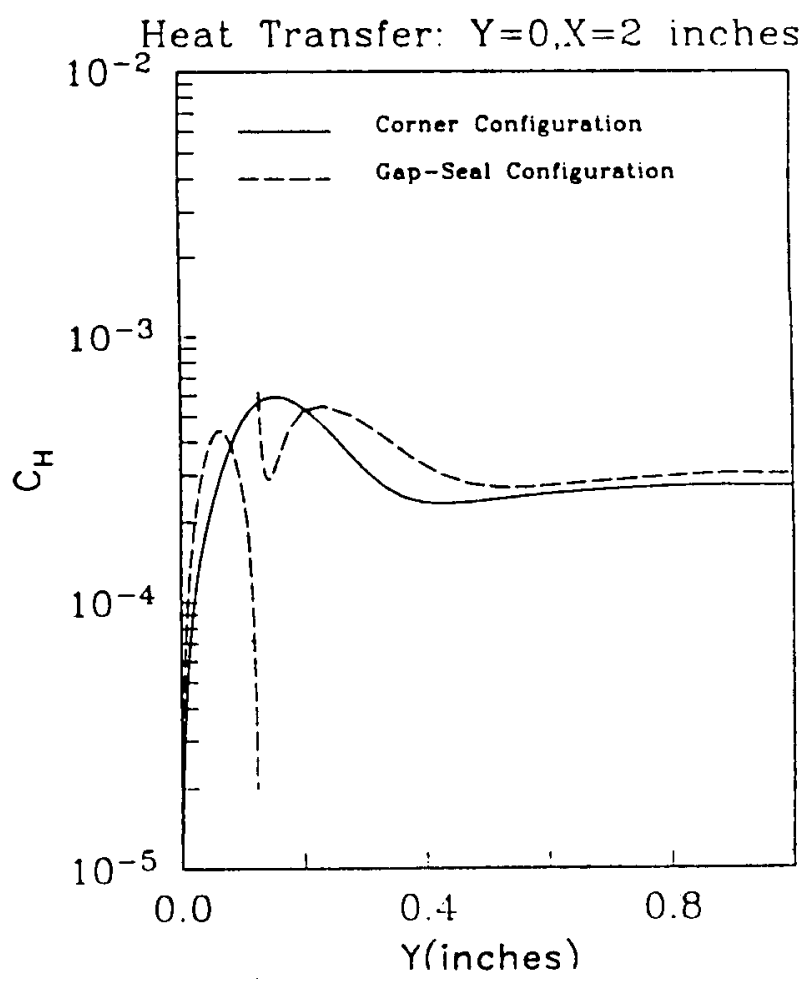

Figure 18 (a)Lateral Variation of Heat Transfer Rates, Corner/Gap seal: $X=2.0$ inches 


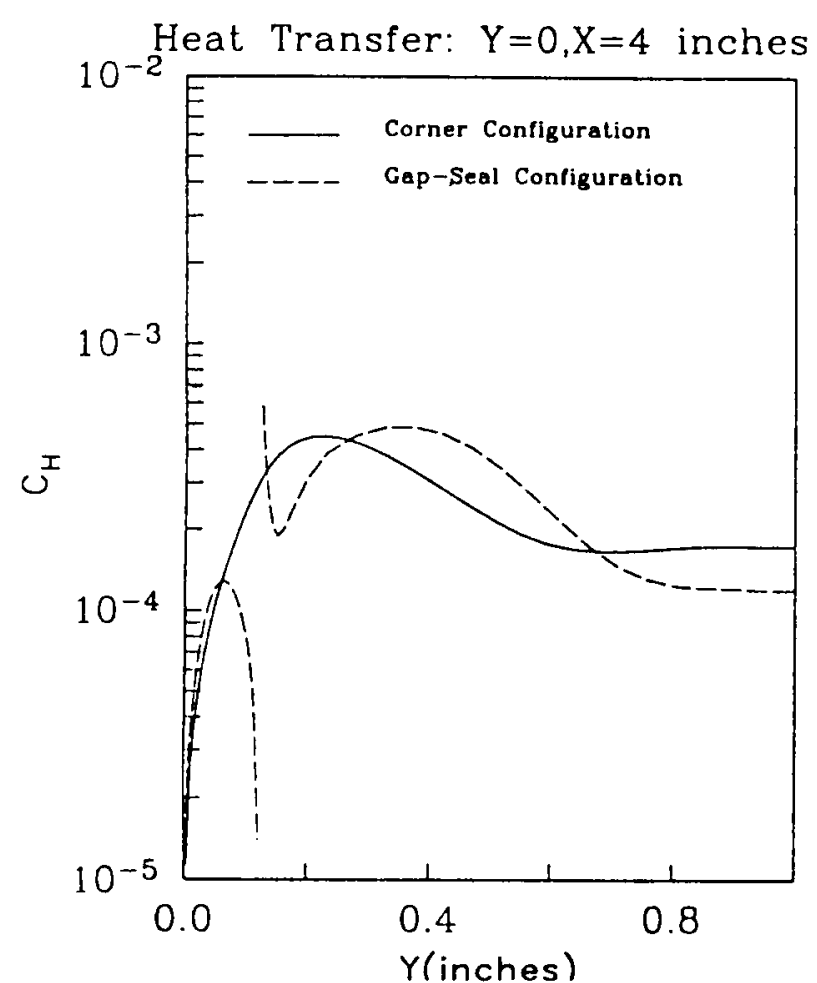

Figure 18 (b)Lateral Variation of Beat Transfer Rates, Corner/Gap seal: $X=4.0$ inches

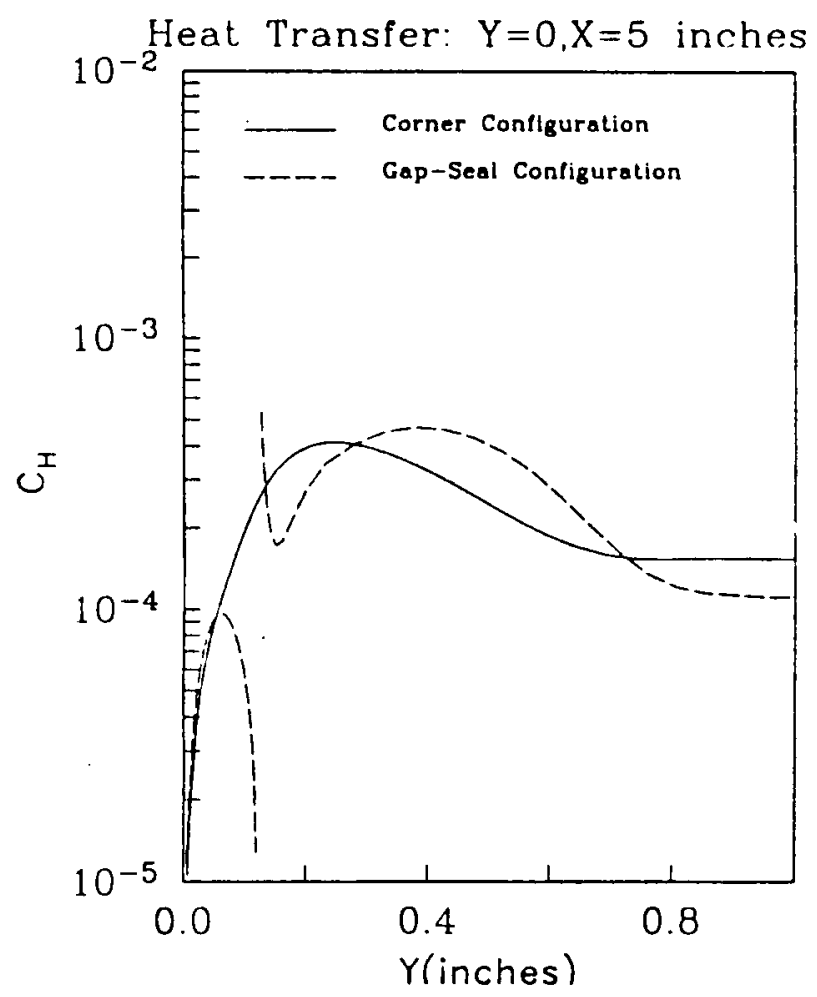

Figure 18 (c)Lateral Variation of Heat Transfer Rates, Corner/Gap seal: $X=5.0$ inches

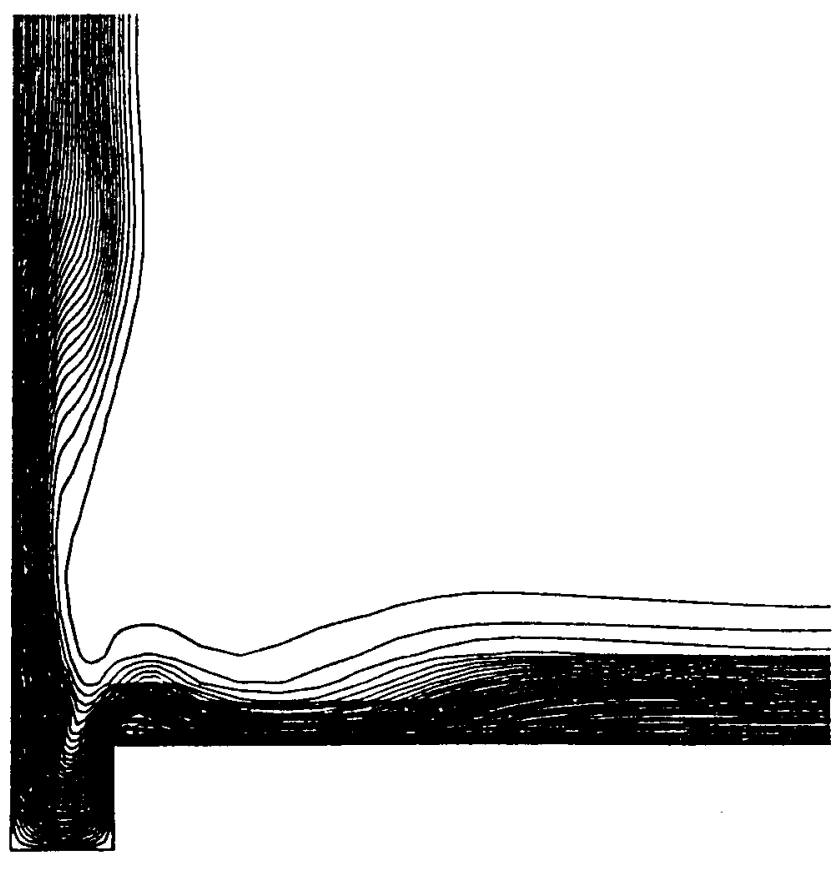

Figure 19 Gap Seal Case: Acial Velocity Component Contours at $X=4.7$ inches. 


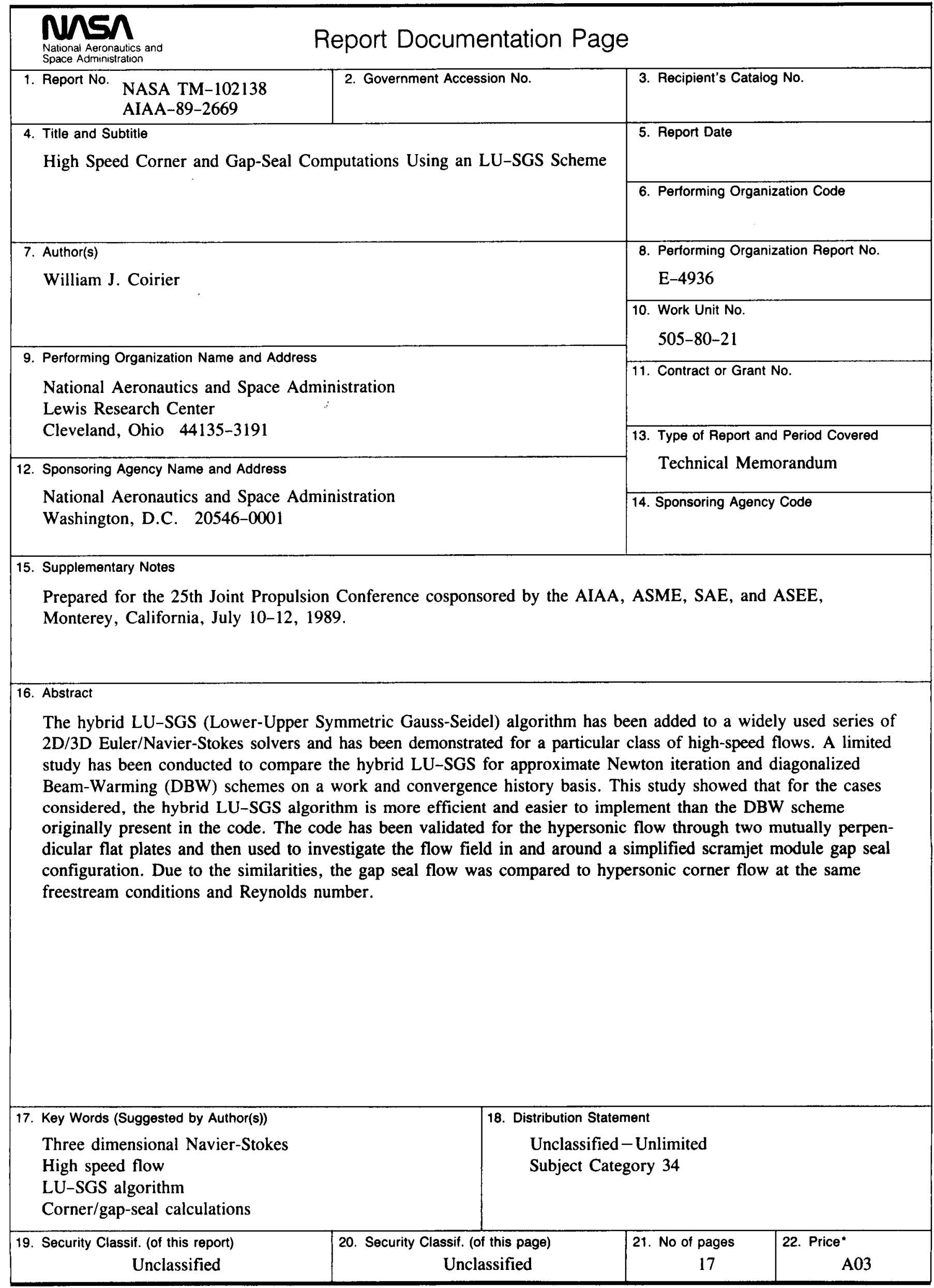

